Journal of

\section{Synchrotron}

Radiation

ISSN 0909-0495

Received 24 October 2008

Accepted 15 January 2009

\title{
Synchrotron-based radioscopy employing spatio-temporal micro-resolution for studying fast phenomena in liquid metal foams
}

\author{
A. Rack, ${ }^{a, b *}$ F. García-Moreno, ${ }^{\text {c,d }}$ T. Baumbach ${ }^{b}$ and J. Banhart ${ }^{c, d}$ \\ ${ }^{\mathbf{a}}$ European Synchrotron Radiation Facility, 38043 Grenoble Cedex, France, ${ }^{\mathbf{b}}$ Forschungszentrum \\ Karlsruhe - ANKA, 76021 Karlsruhe, Germany, ${ }^{\mathbf{c} T e c h n i s c h e ~ U n i v e r s i t a ̈ t ~ B e r l i n, ~} 10623$ Berlin, Germany, and \\ dHelmholtz Centre Berlin (Hahn-Meitner-Institut), 14109 Berlin, Germany. E-mail: arack@snafu.de
}

\begin{abstract}
Investigations of pore coalescence and individual cell wall collapse in an expanding liquid metal foam by means of X-ray radioscopy with spatio-temporal micro-resolution are reported. By using white synchrotron radiation for imaging, the rupture of a film and the subsequent merger of two neighbouring bubbles could be recorded with a time sampling rate of 40000 frames s ${ }^{-1}$ (25 $\mu \mathrm{s}$ exposure time) and a spatial sampling rate of $20 \mu \mathrm{m}$. The rupture time of a cell wall was found to be in the range of $300 \mu$ s. This value is in agreement with theoretical considerations which assume an inertia-dominated rupture time of cell walls in liquid metal foams.
\end{abstract}

Keywords: X-ray radioscopy; metal foams; coalescence; CMOS; micro-radiography; liquid films; metallic thin films; viscosity; fracture; aluminium alloys; bubbles.

\section{Introduction}

With his famous high-speed movies, Lucien Bull impressively showed the outstanding scientific value of time-resolved imaging (Bull, 1928). By using hard X-rays instead of visible light, the application fields of high-speed imaging can be extended to make visible internal structures of opaque objects as they change with time. While even fast periodic movements can be imaged by weak radiation sources via stroboscopic techniques (see e.g. Kardjilov et al., 2005), the actual challenge lies in aperiodic movements that require a high density of photons for imaging. Modern third-generation synchrotron light sources with their insertion devices deliver an X-ray photon flux density high enough to perform radiography with micro-resolution in space and time (Di Michiel et al., 2005; Uesugi et al., 2006), currently only limited by the available detectors and the time structure of the pulsed synchrotron radiation. Imaging of triggered events using a single light pulse from an undulator source has already been reported (Wang et al., 2008).

In this paper we introduce high-speed X-ray image sequences ('movies') captured with an image repetition rate of 40000 frames s ${ }^{-1}$ (FPS), which equals $25 \mu \mathrm{s}$ exposure time. The movies image continuously the temporal evolution within a foaming metal over a time interval of several seconds. First applications of micro-radiography with a moderate acquisition speed of around 1 FPS to study in situ metal foaming showed the enormous potential of the method as well as a demand for higher acquisition rates (Banhart et al., 2001). To measure the rupture time of a cell wall and to access the properties of the constitutive liquid metal in a single film would allow for a better understanding of the stabilization mechanisms. A recent experiment gave indication that highest acquisition rates above 10000 FPS are required for that (García-Moreno et al., 2008).

\section{Experiments}

Experiments with white synchrotron radiation were carried out at the high-flux beamline ID15a of the European Synchrotron Radiation Facility (ESRF), France (Di Michiel et al., 2005). The X-ray pixel detector used is based on the indirect detection concept as introduced for live topography (Hartmann et al., 1975): a scintillator screen converts X-ray photons into visible light. The resulting luminescence image is optically coupled to a digital camera. In our case, the optical system is an ESRF in-house development allowing for a 1:1 projection of the screen onto the camera (Koch, 1994). As scintillating material, commercially available bulk LuAG:Ce (200 $\mu$ m thick) was chosen which is known to be suited for fast synchrotron-based imaging involving high heat loads (Di Michiel et al., 2005; Touš et al., 2008; Banhart, 2008). The white beam of the ID15a insertion device was filtered with $25 \mathrm{~mm}$ of silicon, leading to an X-ray photon flux density in the range of $10^{15}$ photons $\mathrm{s}^{-1} \mathrm{~mm}^{-2}$ (ESRF, 2009). For high-speed data acquisition the novel CMOS camera Photron Fastcam SA-1 was applied (Inoue et al., 2005). The CMOS chip with $1024 \times 1024$ pixels (each $20 \mu \mathrm{m}$ in size) has a peak quantum efficiency of $42 \%$ at $640 \mathrm{~nm}$ and a ten-bit dynamic range (800:1). One signal unit (ADU) corresponds to a charge of 5.5 electrons in the corresponding potential well of the chip. The camera can acquire 5400 FPS in full-frame mode and up to 675000 FPS when using a region of interest (ROI). The minimal shutter time is $2 \mu \mathrm{s}$; the shutter can be triggered with a time resolution of $100 \mathrm{~ns}$. The images as read out are stored in a 32 Gbyte onboard memory which defines the maximum recording length. The movies we show are taken with a ROI to reach 40000 FPS ( $25 \mu$ s exposure time) and an effective pixel size of $20 \mu \mathrm{m}$ (leading to a spatial resolution $R>40 \mu \mathrm{m}$ ).

Aluminium-based foamable compacts were made following the powder metallurgical route, i.e. by mixing the elemental metal 
powders with $\mathrm{TiH}_{2}$ acting as blowing agent and pressing these. The precursors were foamed inside a furnace pressurized with argon gas. It consisted mainly of an $\mathrm{AlMg} 1$ tube $(40 \mathrm{~mm}$ diameter and $0.5 \mathrm{~mm}$ wall thickness) with a ceramic heating plate inside. The furnace has already been described in more detail previously (García-Moreno et al., 2005). The coalescence rate during foaming under normal conditions is usually in the range of some events per second in a sample of the size used. To accelerate expansion during the short available time window (a few seconds) and to provide more observable coalescence events, we depressurized the furnace during expansion. Owing to fast expansion of the foam during pressure release, a large number of coalescence events could be recorded.

In summary, the foaming procedure comprised three steps: (i) heating of the precursor under 5 bar pressure, (ii) melting of the precursor and nucleating pores still under pressure, and (iii) fast pressure release from 5 bar to 1 bar, thereby triggering fast foam expansion.

\section{Results and discussion}

Fig. 1 shows a sequence of images, each of which are five or more frames apart, corresponding to $125 \mu$ s or more. To demonstrate the features that can be seen much more clearly in the moving image, ${ }^{1}$ the right-hand column shows the silhouette of two bubbles in the foam that merge to one larger bubble in various stages. (i) The film separating the two bubble is still intact, see Fig. 1(a). (ii) The film ruptures and is absorbed into the films roughly perpendicular to it, see arrows in Fig. 1(b). (iii) The merging dumbbell-shaped twin bubble flattens during this absorption (Fig. 1c). The films into which the obsolete film has been sucked are flat $12 \pm 3$ frames $(300 \pm 75 \mu \mathrm{s})$ after the initiation of rupture [this has been termed 'rupture time' previously by García-Moreno et al. (2008)]. (iv) The two bubbles move together and form a near-spherical unique bubble after another $900 \pm 50 \mu \mathrm{s}$. (v) The bubble continues to oscillate slowly for some time (Figs. $1 d$ and $1 e$ ).

The rupture time measured, $300 \mu$ s, can be compared with the larger value of $600 \mu$ s obtained by García-Moreno et al. (2008). That value corresponded to a bubble with $3 \mathrm{~mm}$ diameter, and a lower value is expected here since the rupture time is proportional to the diameter within the simple model presented by García-Moreno et al. (2008). Hence, as the diameter of the two bubbles before merger is $\sim 1.4 \mathrm{~mm}$ and $\sim 1.1 \mathrm{~mm}$, and that of the merged bubble is $\sim 1.6 \mathrm{~mm}$, the rupture time measured appears to be in the range expected. This finding underlines that the rupture of a single cell wall in a liquid metal foam is dominated by inertia. The oxide particles present in the liquid metal do not prevent the moving metal from being very fluid although they form a network penetrating the entire film (Dudka et $a l ., 2008)$. One possible explanation is that the network is disrupted when a film ruptures, during which the apparent melt viscosity sharply drops (García-Moreno et al., 2008). This also implies that the viscosity of the melt is not what makes a melt foamable as is often stated (see, for example, Song et al., 2000).

In conclusion, we could measure a fast phenomenon in liquid metal foam via synchrotron-based radioscopy with spatio-temporal microresolution. The technique will allow for the investigation of film rupture in dependence of, for example, alloy composition, content of solid particles, temperature or pore radius. The current experiments only used a fraction of the photon flux density available as the high

\footnotetext{
${ }^{1}$ Supplementary data for this paper are available from the IUCr electronic archives (Reference: KV5057). Services for accessing these data are described at the back of the journal.
}
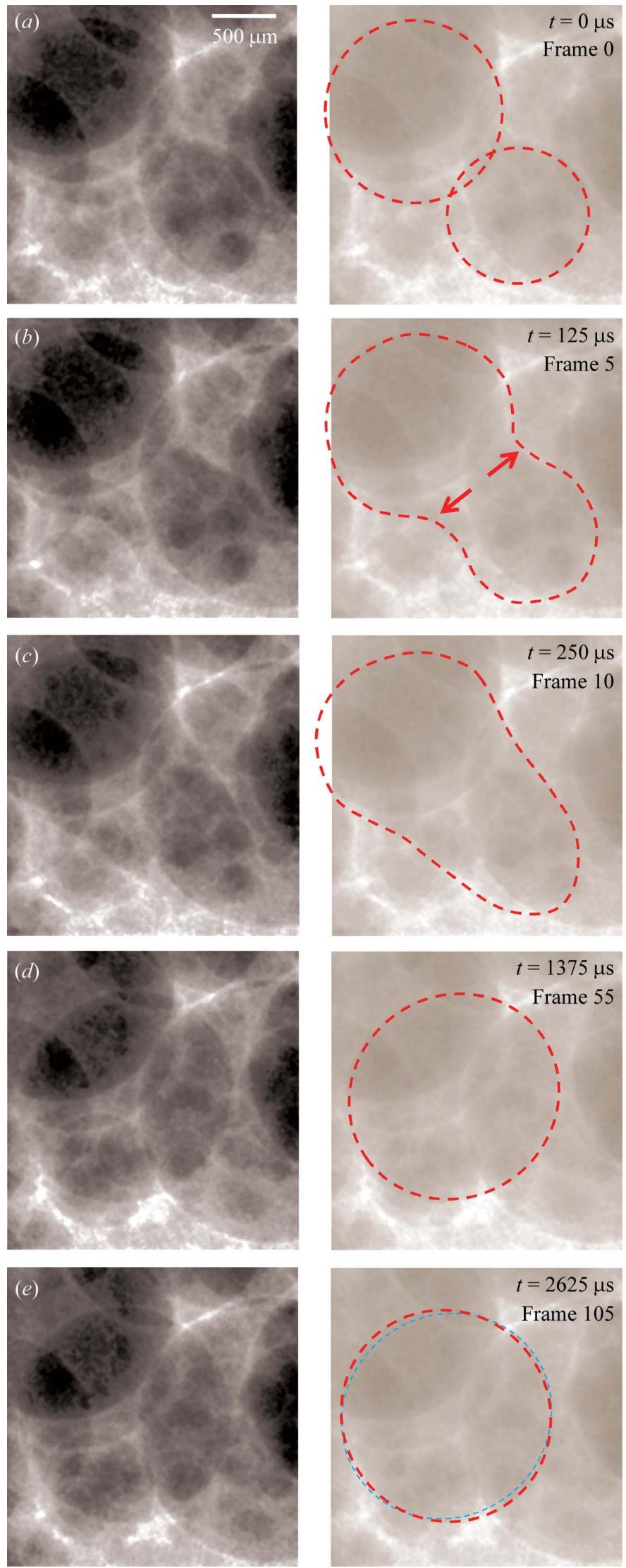

Figure 1

Left column: selected images of a series of 184000 showing film rupture in $(a)$ to $(c)$ and bubble oscillation in $(d)$ and $(e)$. Right column: features discussed in the text. 


\section{short communications}

heat load of the source on the scintillating screen otherwise would irreversibly damage it. Thus, with the ongoing detector development, frame rates above 100000 FPS will soon be feasible.

T. Martin, C. Jarnias, M. Peele, M. Di Michiel (ESRF) and T. dos Santos Rolo, D. Haas, A. Ershov, L. Helfen (ANKA) contributed to the success of this experiment.

\section{References}

Banhart, J. (2008). Advanced Tomographic Methods in Materials Research and Engineering. Oxford University Press.

Banhart, J., Stanzick, H., Helfen, L. \& Baumbach, T. (2001). Appl. Phys. Lett. 78, 1152-1154.

Bull, L. (1928). La Cinématographie. Paris: Armand Collin

Di Michiel, M., Merino, J. M., Fernandez-Carreiras, D., Buslaps, T., Honkimäki, V., Falus, P., Martin, T. \& Svensson, O. (2005). Rev. Sci. Instrum. 76, 043702.
Dudka, A., García-Moreno, F., Wanderka, N. \& Banhart, J. (2008). Acta Mater. 56, 3990-4001.

ESRF (2009). ID15 High Energy Diffraction and Scattering Beamlines, http:// www.esrf.Fr/UsersAndScience/Experiments/MaterialsScience/ID15/.

García-Moreno, F., Babcsán, N. \& Banhart, J. (2005). Colloids Surf. A, 263, 290-294.

García-Moreno, F., Rack, A., Helfen, L., Baumbach, T., Zabler, S., Babcsán, N., Banhart, J., Martin, T., Ponchut, C. \& Di Michiel, M. (2008). Appl. Phys. Lett. 92, 134104.

Hartmann, W., Markewitz, G., Rettenmaier, U. \& Queisser, H. J. (1975). Appl. Phys. Lett. 27, 308-309.

Inoue, T., Takeuchi, S. \& Kawahito, S. (2005). Proc. SPIE, 5580, 293-300.

Kardjilov, N., Hilger, A., Manke, I., Strobl, M., Treimer, W. \& Banhart, J. (2005). Nucl. Instrum. Methods Phys. Res. A, 542, 16-21.

Koch, A. (1994). Nucl. Instrum. Methods Phys. Res. A, 348, 654-658.

Song, Z.-L., Ma, L.-Q., Wu, Z.-J. \& He, D.-P. (2000). J. Mater. Sci. 35, 15-20.

Touš, J., Horváth, M., Pína, L., Blažek, K. \& Sopko, B. (2008). Nucl. Instrum. Methods Phys. Res. A, 591, 264-267.

Uesugi, K., Sera, T. \& Yagi, N. (2006). J. Synchrotron Rad. 13, 403-407.

Wang, Y., Liu, X., Im, K.-S., Lee, W.-K., Wang, J., Fezzaa, K., Hung, D. L. S. \& Winkelman, J. R. (2008). Nat. Phys. 4, 305-309. 